\title{
PERCEPOLIS: Pervasive Cyberinfrastructure for Personalized Learning and Instructional Support*
}

\author{
Ali R. Hurson, Sahra Sedigh \\ Missouri University of Science and Technology, Rolla, USA \\ E-mail: \{hurson,sedighs\}@mst.edu \\ Received June 11, 2010; revised August 23, 2010; accepted October 2, 2010
}

\begin{abstract}
This paper describes PERCEPOLIS, an educational platform that leverages technological advances, in particular in pervasive computing, to facilitate personalized learning in higher education, while supporting a networked curricular model. Fundamental to PERCEPOLIS is the modular approach to course development. Blended instruction, where students are responsible for perusing certain learning objects outside of class, used in conjunction with the cyberinfrastructure will allow the focus of face-to-face meetings to shift from lecture to active learning, interactive problem-solving, and reflective instructional tasks. The novelty of PERCEPOLIS lies in its ability to leverage pervasive and ubiquitous computing and communication through the use of intelligent software agents that use a student's academic profile and interests, as well as supplemental information such as his or her learning style, to customize course content. Assessments that gauge the student's mastery of concepts are used to allow self-paced progression through the course. Furthermore, the cyberinfrastructure facilitates the collection of data on student performance and learning at a resolution that far exceeds what is currently available. We believe that such an infrastructure will accelerate the acquisition of knowledge and skills critical to professional engineering practice, while facilitating the study of how this acquisition comes about, yielding insights that may lead to significant changes in pedagogy.
\end{abstract}

Keywords: Learning Technology, Personalized Learning, Cyberinfrastructure, Pervasive Computing

\section{Introduction}

For the past 800 years, educational systems have relied heavily on a traditional course delivery environment, which has a lecture-based (one-instructor-to-many students) model [1] and a discrete, linear course structure. Advances in databases, distributed computing, computational intelligence, and especially pervasive computing, which allows transparent, non-intrusive, "anytime, anywhere" access to information, provide fertile ground for radical changes in pedagogy and instructional design.

In Educating Engineers: Designing for the Future of the Field [2], direct observation of forty schools of engineering and examination of 100 accreditation self-study reports serve as the basis for evaluating the state of undergraduate engineering education in the United States.

One of the principle findings of this report was that undergraduate engineering education is focused on the

*This work was supported in part by the U.S. National Science Foundation under Grant No. IIS-0324835. transfer of technical knowledge to the students, rather than on preparing them for professional practice. The report defines practice as "the complex, creative, responsible, contextually grounded activities that define the work of engineers at its best," and professional as "those who can be entrusted with responsible judgment in the application of their expertise for the good of those they serve."

Another important finding of the report was the "linearity" of the dominant curricular model, which is characterized as fundamentally contradictory to the body of knowledge on how students learn. To address this shortcoming, a networked curricular model has been proposed, where the components form a cohesive and strongly interconnected whole in which learning in one area reinforces and supports learning in other areas. The trajectory followed by each student through the curriculum can and should differ, based on prior knowledge and skills, learning styles, and interests of the student, among other attributes. The importance of personalizing this 
trajectory is underscored by the selection of personalized learning as one of fourteen Grand Challenges for Engineering identified by the National Academy of Engineering [3].

The platform described in this paper rises to the challenge by leveraging technological advances, in particular in pervasive computing, to create a cyberinfrastructure that facilitates personalized eLearning in engineering education, while supporting a networked curricular model. A plethora of enabling technologies, including intelligent software agents, distributed and heterogeneous databases, and global information sharing processes are leveraged to develop Pervasive $\underline{\text { Cyberinfrastructure }}$ for Personalized Learning and Instructional Support (PERCEPOLIS). We believe that this infrastructure will accelerate the acquisition of knowledge and skills critical to professional engineering practice, while facilitating the study of how this acquisition comes about, yielding insights that may lead to significant changes in pedagogy.

Fundamental to PERCEPOLIS is the modular approach to course development, which increases the resolution of the curriculum and allows for finer-grained personalization of learning processes and associated data collection. Each course is composed of several content modules, a number of which are mandatory, as dictated by course and curriculum objectives. The remaining content modules, typically smaller in number, include elective content that can be chosen to supplement the student's knowledge of prerequisites, or to engage an interested student in more advanced topics. The content modules for each course can be supplemented by experiential modules, which are intended to reemphasize key issues covered in the content modules through hands-on individual and group projects. The experiential modules are dynamic, as their contents can be determined based on topics that a majority of the students in a given class find challenging or confusing.

Associated with each module are learning objects such as prerequisite modules, lecture notes, problems, sample solutions, and programming or laboratory exercises. Modules in different courses can be linked to each other, facilitating implementation of a networked curricular model. Furthermore, the blended learning approach, where students are responsible for perusing certain learning objects outside of class, allows the focus of face-to-face meetings to shift from lecture to active learning, interactive problem-solving, and reflective instructional tasks.

As depicted in Figure 1, the proposed cyberinfrastructure is in the form of middleware that links databases, e.g., PeopleSoft, and instructional platforms, e.g., Blackboard, Angel, currently in use at academic institu- tions, facilitating incremental adoption of the networked and modular curricular model at low cost. Moreover, the ability to connect tools already in use, without requiring customized computing resources, enables instructors at different academic institutions to collaborate on and exchange educational materials and data, creating an environment conducive to collaboration on educational research.

\section{Background and Enabling Technologies}

PERCEPOLIS enables effective and personalized learning while taking advantage of current advances in hardware/software technology; generates a wealth of information that can be analyzed to understand how students learn; facilitates the enrichment of engineering and science curricula with missing basics such as interpersonal skills; encourages proactive, robust, dynamic, and continuous development of courses and networked curricula; allows affordability, availability, and accessibility of higher education; reduces inter- and intracurriculum redundancy; facilitates reusability of curricula and courses; promotes proactive and interactive learning; reduces the student-to-faculty ratio; and provides greater accessibility and transparency to curriculum and course contents via user-friendly navigational tools built on top of existing information management systems.

We rely on our research in the areas of pervasive computing, mobile agent technology, and global information sharing to achieve these objectives. Pervasive computing exploits the increasing applications and capabilities and declining costs and size of computing and communications devices to provide continuous, nonintrusive, and automated services to a growing number of domains, including education, defense, and industry $[4,5]$. In pervasive computing, computers work in the background to proactively and autonomously, based on the context, provide temporal and spatial information and services to stationary or mobile users, necessitating the use of intelligent entities, as well as cooperation among the devices or systems involved. Pervasive computing has the potential to significantly improve the handling of dynamic situations and exceptions in disparate environments, through the use of software agents that monitor and record events and take proactive and collaborative action to ensure the availability of relevant information in an adaptive fashion. The adaptability and flexibility of pervasive computing can facilitate a radical change in the way students interact with professors and peers.

An agent is a computer program that acts autonomously on behalf of a person or organization [6]. A mobile agent 


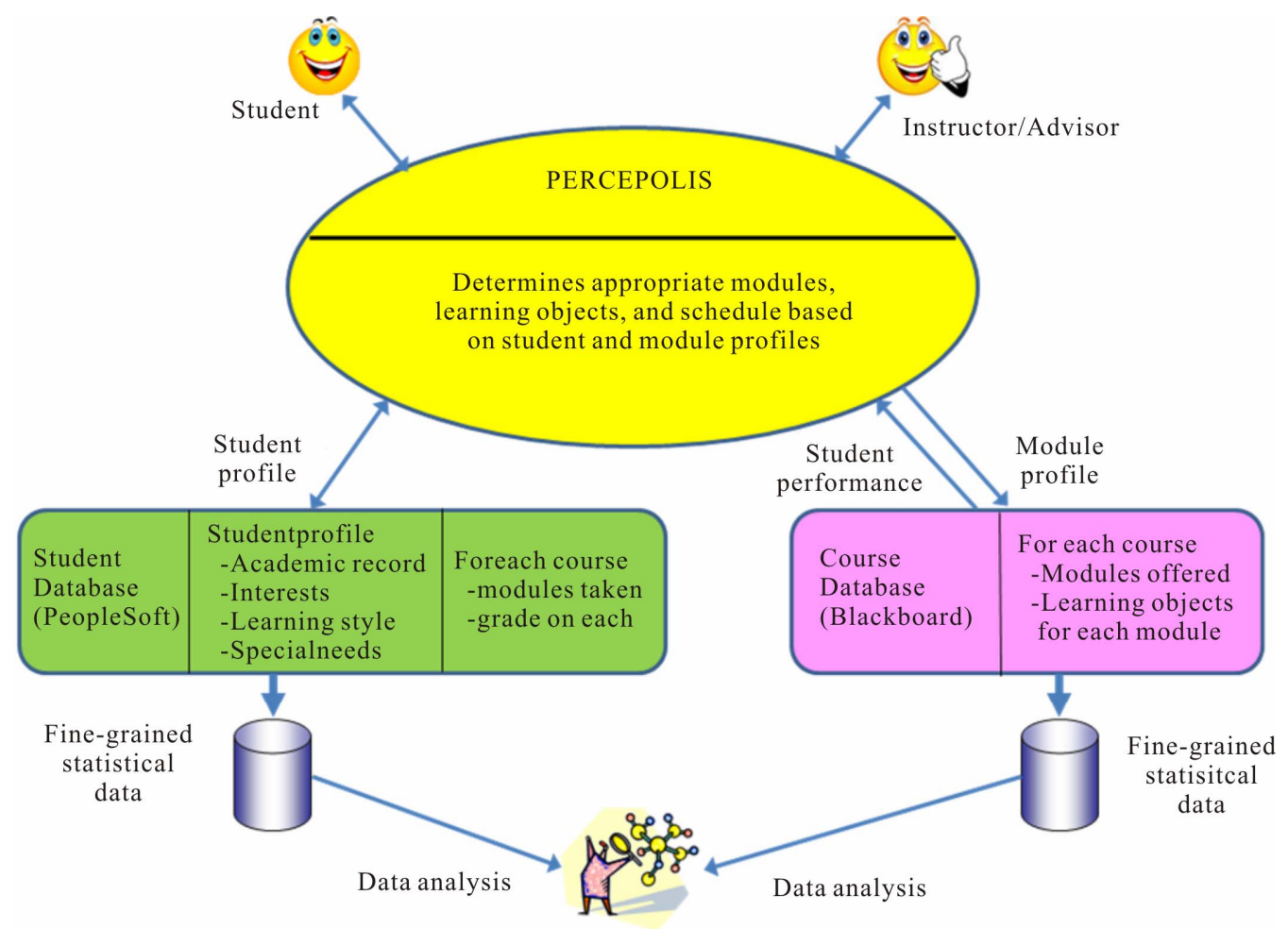

Figure 1. Overview of PERCEPOLIS cyberinfrastructure.

moves through heterogeneous networks autonomously, migrating from host to host, and interacting with other agents [7]. Agent-based distributed application design is gaining wider acceptance, as it provides an efficient and robust framework for tasks ranging from information retrieval to the administration of complex distributed systems [8]. Agents are also used in environments where intelligent software can help increase performance and reliability; such scenarios are evident in the use of agents in search and tracking applications. The advantages of mobile agent technology in supporting disconnected operation, load balancing, and reducing network traffic in global information sharing have been extensively studied in the literature. As an example, a quantitative study of the effect of mobile agents on reducing communication cost, improving response time, and conserving energy is reported in [9].

Our previous work includes the application of mobile agents within the scope of the mobile data access systems and dynamically-configured sensor networks [8], [10-14]. Especially relevant to the proposed cyberinfrastructure is the development of a prototype mobile data access system that demonstrates the suitability and robustness of mobile agent technology in a global information sharing environment characterized by limited network bandwidth, power, and computational resources; and weak and unreliable network connectivity.

The novelty of PERCEPOLIS lies in its ability to exploit pervasive computing and communications at various levels, through the use of the agent-based middleware developed in our previous work [8]. The software agents can be launched by a user (student or instructor/advisor), an application (course scheduling, advising), or another agent. Agents work diligently on behalf of their creator, sharing information and resources with other agents. In this context, the developed middleware creates mission-oriented dynamic computing entities that autonomously perform tasks on behalf of users, and provides a framework for adaptively composing an appropriate curriculum. The proposed framework supports a networked curricular model and in the process, enriches course delivery, improves the quality of course content, and encourages interaction. Mobile agents allow for more dynamic and robust implementation of the artifacts developed.

The recent exponential growth of information on the web, advances in information technologies, wireless communication capabilities, and Internet access devices necessitates a change to the traditional notion of timely and reliable access to information. It is believed that for many application domains, the Internet contains much of the needed information, in some form or shape, and is capable of delivering it at high speeds. However, the information may not be available in the form required by the user. In other words, accessibility (rather than availability) of information is the main challenge. What is 
needed are means to: 1) locate relevant information intelligently, efficiently, and transparently; 2) extract, process, and integrate relevant information effectively; and 3) interpret the processed information intelligently.

Our research within the scope of global information sharing, multidatabases, and multimedia data processing has resulted in the design and implementation of an intelligent search engine, denoted as the Summary Schemas Model (SSM) [15-19], that supports automatic identification of semantically similar/dissimilar data that have different/same names and representations in a wired or wireless platform. The model uses word relationships, i.e., hypernym, hyponym, synonym, defined in a standard thesaurus such as Roget's, to automatically build hierarchical metadata of local access terms exported from underlying local databases.

The SSM model has been simulated and a prototype architecture has been developed, supported in part by funding from DARPA. The performance of the model has been evaluated under various schema distributions, query complexities, and network topologies. The simulation results showed that both precise and imprecise queries incur comparable costs, and hence yield compatible performance. In certain cases, the SSM imprecise query processing even outperformed precise query processing $[16,20]$. The simulator also showed the dynamic nature of SSM, by allowing incremental addition of nodes (databases) without change to other databases and with minimal interruption.

Within the scope of the SSM, we have also investigated issues such as query/transaction processing, and security. The scope of the SSM was extended to support mobility, resulting in development of the mobile data access system (MDAS), which enables representation and manipulation of multimedia information $[9,10,18]$. In general, the SSM and its successor, MDAS, preserve local autonomy, high expandability and scalability, short response time, and resolution of imprecise queries. In separate research [21], we demonstrated the capability to link SSM to commercially available search engines. This feature is of special relevance to the proposed cyberinfrastructure, as it enables students and instructors to automatically explore databases and resources external to the entities formally associated with the project.

We use our research experiences in the area of multidatabases [22], mobile data access systems [19,23], and mobile agent technology [9-14] to develop:

- A semantic network (where entities are interconnected based on their semantics) and semantic metadata that integrates course modules within and across curricula (networked curriculum).

- A navigational tool that enables a student to follow a personalized trajectory of modules for each course, which has been determined based on information including his/her academic profile, interests, learning style, and special needs. Moreover, the same navigational tool can be used by advisors to facilitate course selection, as described in Section 3.

Putting it all together, in PERCEPOLIS, course modules are stored as a heterogeneous database populated by learning objects in a variety of formats and media. The middleware will provide an easy interface to the course material for both on-campus and distance students, making anytime, anywhere learning a reality.

\section{Design of the Cyberinfrastructure}

The PERCEPOLIS platform views a degree-granting program as three sets of entities: 1) the set of instructors/advisors, $I$; 2) the set of students, $S$; and 3) the set of courses, $C$. An instructor/advisor, $i \in I$, has expertise in one or more subjects. A student, $s \in S$, is studying towards a degree and is required to take courses from $C$, in an orderly fashion, to satisfy degree requirements and objectives. Each $c \in C$ represents a course in the curriculum. The courses in $C$ are interrelated, per the structure of the curriculum. In addition, courses are tagged as required or elective, according to degree requirements. Each of $I, S$, and $C$, respectively, is represented by a community of software agents that communicate and negotiate with each other according to the defined tasks, e.g., personalizing the trajectory through a course or curriculum. As described in Section 3.1, we use a modular approach in the development and delivery of courses, so that each course $c \in C$ can be viewed as a collection of interrelated modules, where the degree of connectivity among modules can vary from course to course.

\subsection{Modular Course Development}

Fundamental to the proposed educational platform is the modular approach to course development. Each course is divided into several content modules, a number of which are mandatory, as dictated by the curriculum to satisfy degree objectives, requirements, and accreditation requirements, e.g., ABET. The remaining content modules, typically smaller in number, include elective content that can be chosen to supplement the student's knowledge of prerequisites, or to engage an interested student in more advanced topics. The content modules for each course can be supplemented by experiential modules, which are intended to reemphasize key issues covered in the content modules through hands-on individual and group projects. The experiential modules are dynamic, as their contents can be determined based 
on topics that a majority of the students in a given class find challenging or confusing. Associated with each module are learning objects such as prerequisite modules, lecture notes, problems, sample solutions, and programming or laboratory exercises.

Course modules are self-contained, self-paced, and designed to promote active rather than passive learning. Available multimedia tools are used to design a module to be interactive and flexible for the students, who can navigate freely through the program. Self-assessment questions at the end of each module will take a variety of forms, including objects to be "dragged" into place, and multiple-choice or short-answer questions. The most interactive learning objects of a course module are the design challenges, in which the student must make decisions and respond to "hints" and other feedback in order to find a solution for the problem at hand. Graphics and animation are used to demonstrate complex concepts. Text, narration, charts, and diagrams are used to reinforce concepts during animation.

All courses are "mix-and-match," in the sense that each consists of several modules on interrelated topics which are, in turn, interrelated across the whole curriculum in a networked fashion. Figure 2 illustrates a sample environment for a course on design and analysis of algorithms. In this example, a module on graphs in the algorithms course is closely linked to other modules of the same course - sorting, greedy algorithms, dynamic programming, and complexity analysis. Furthermore, the module representing routing in a course on computer networks is linked to the graphs module of the algorithms course.

Some modules require prior knowledge; if the student does not have this background, he/she should be able to peruse a module from an earlier course before reentering the current module. Assessments that gauge the student's mastery of concepts at the module level are used to allow self-paced progression through the course. If a student has already mastered the material in a module and wishes to explore the topic in more depth, he/she can choose a more advanced module in that subject. To maintain a sense of community, advanced students who progress through the course more rapidly are encouraged to serve as group leaders. This peer learning approach fosters "soft" skills, such as communication and negotiation. Figure 3 depicts the possible trajectories of a student through one module of a course. The figure also demonstrates the student tracking process as incorporated in the module. Each module reinforces background knowledge and allows self-paced progression and options to study more advanced concepts and/or to gain missing basics.

In short, modular course design offers:
- Adaptability/flexibility: to a certain extent, course contents can be personalized based on a student's needs and interests. This is particularly beneficial to students in honors or certificate programs, graduate students, and transfer students.

- Continuity: the modular approach to the course design, combined with the ability to personalize the contents of a course allows greater continuity in the curriculum, while satisfying the needs of some students without sacrificing the needs of others.

\subsection{Blended Instruction and Learning}

Common experience shows that online availability, e.g., through a course management system such as Blackboard, of lecture notes and other instructional content generally leads to low class attendance by students. Even students who continue to regularly attend classes may become inattentive in the classroom and passive in any discussions that take place. Furthermore, there is an increasing expectation that the course coverage and examination must be limited to whatever is available online, and any topics discussed outside of class should not be covered on the tests. Periodic examinations and pop quizzes (though extremely unpopular among the students), to some degree, could be reasonable solutions; but are becoming scarce due to large class sizes. We seek to adopt a new teaching practice that is attuned to advances in technology, is interactive and motivates active class participation, enforces continuous learning, and fosters interpersonal skills that may be neglected in a traditional classroom. To achieve these objectives, we propose to modify the traditional lecture-based teaching environment, by utilizing a blended approach to learning and instruction, with the following features:

- Most, if not all, of the class period is dedicated to interactive problem-solving based on a collection of course materials covered by modules discussed in current and previous classes.

- Students are required to peruse specified learning objects before class. All content modules are made available online in advance, and group study (facilitated by the experimental modules) is highly encouraged.

- Students are expected to either communicate major issues with the instructor ahead of time, bring their questions to the class, and/or use online communication tools such as discussion boards to share their concerns and questions with their peers.

- The class period then is dynamically organized to address major issues raised by students and enhance their understanding of subject matter learned in the content module. 
- To encourage independent learning and reflection and continuous involvement, at the beginning of each class period, students are quizzed on the major conceptual topics (mandatory modules) they have been asked to study. The remainder of the session is devoted to addressing students' questions, and discussing and analyzing the more complex topics covered in the mandatory modules.

- Traditional periodic homework assignments, projects, and examinations will continue to be utilized to reinforce learning and determine the students' overall mastery of course contents. Naturally, implementational details such as absences due to extenuating circumstances need to be addressed in a fair and appropriate fashion.

- Maintain a sense of community, advanced students who progress through the course more rapidly will serve as group leaders and are rewarded with extra credit for their efforts in mentoring and tutoring their peers.

- Finally, provisions are made to encourage, promote, and reward group activities and missing basics such as leadership, communication skills, and ethics.

In short, our perspective of the classroom is an envi- ronment conducive to experimentation and discovery, which can be tailored to cover key issues in the content modules, and more specifically, the mandatory portion of the content modules.

\subsection{Intelligent Access Tools}

We leverage our research in multidatabases, mobile agent technology, pervasive computing, and mobile data access systems to develop middleware that serves as global information sharing cyberinfrastructure. This middleware is positioned on top of existing database and course management systems, and allows anytime, anywhere intelligent and transparent access to content modules that comprise the courses of a curriculum. The embedded dynamic nature and bottom-up design approach of the SSM makes it particularly suitable for extending the scope of PERCEPOLIS beyond a single discipline or educational unit. The summary schemas concept underlies a semantic network and semantic metadata that integrate course modules, and hence courses, within and across multiple curricula.

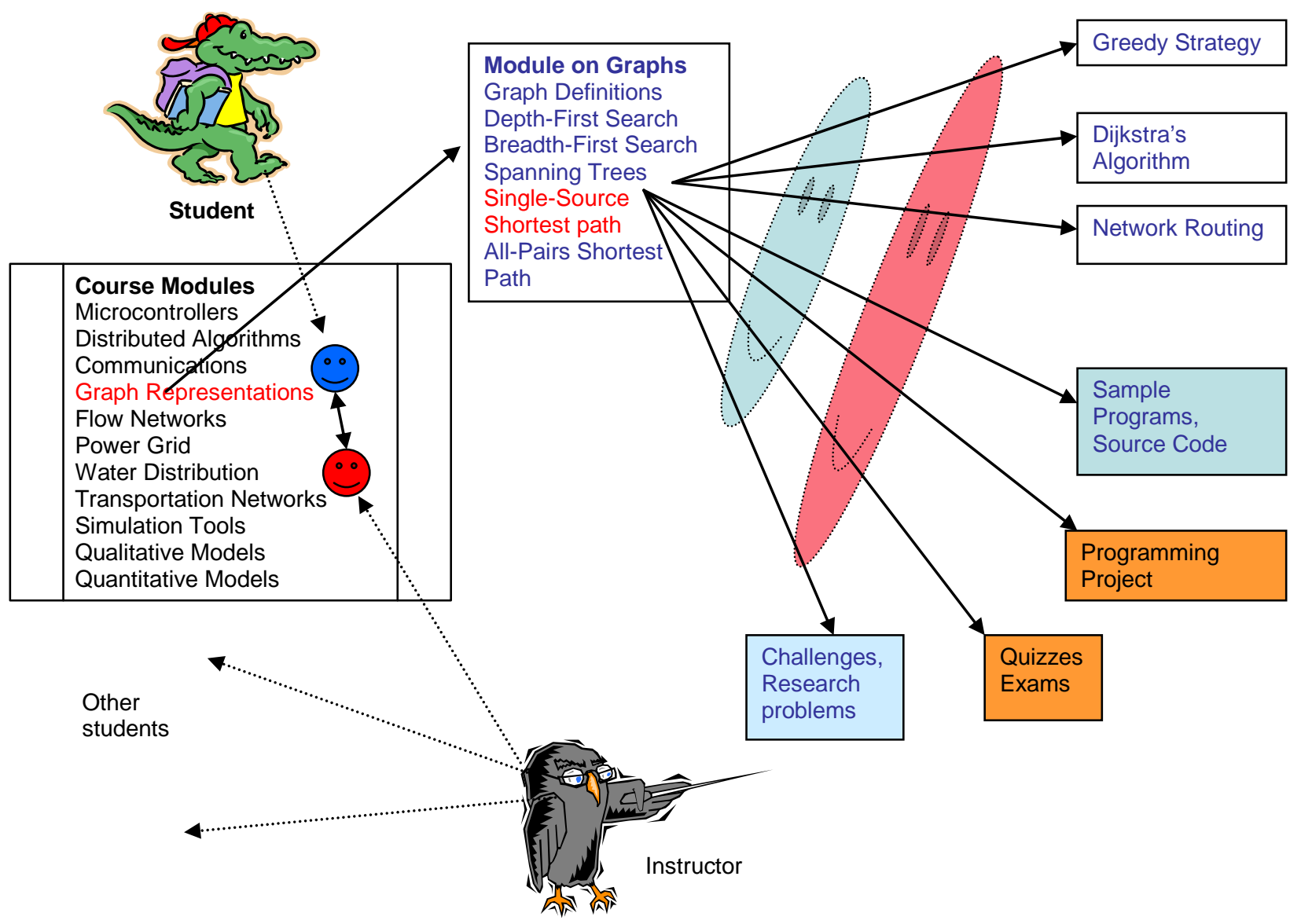

Figure 2. Sample environment for a course on design and analysis of algorithms. 


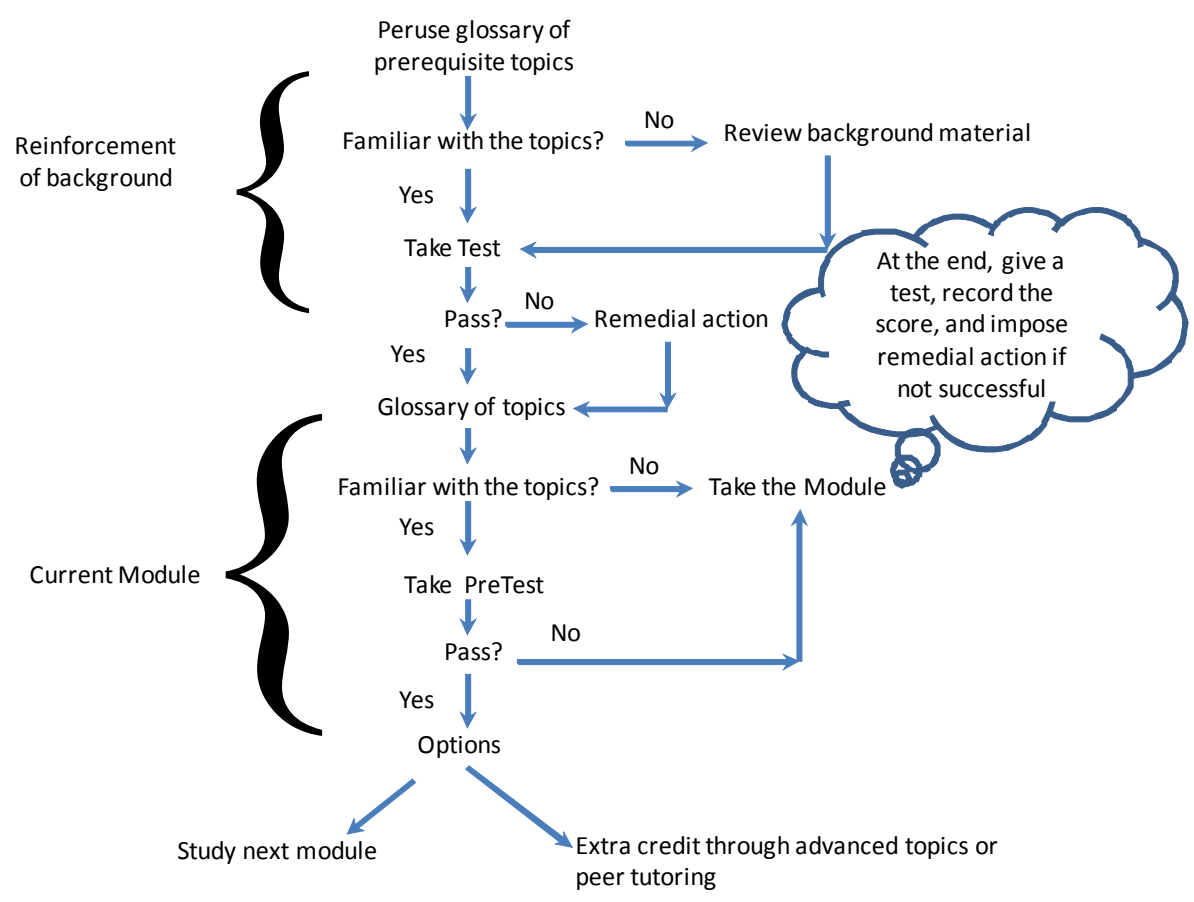

Figure 3. Possible trajectories through one module of a course.

Furthermore, with the aid of the navigational tools and imprecise content-based search capability of the SSM, users, e.g., students, instructors, and advisors, can browse through content modules from a range of courses and curricula to determine an appropriate learning trajectory. The choice of the SSM, regardless of our familiarity with the concept and access to its prototype, stems from: 1) its ability to perform semantic search and imprecise queries, 2) the relatively small size of its metadata, 3) the robustness of the model, 4) its automatic generation of metadata, and finally, 5) the efficiency of the platform.

In the context of PERCEPOLIS, the SSM metadata hierarchy, at the root level, represents the curriculum, e.g., computer science and computer engineering curricula, with semantic terms that specify curriculum objectives and content requirements. The leaf nodes then represent the course modules. The synonym, hypernym, and hyponym links are used to relate modules to courses and/or areas, as defined by the curriculum.

The meta-data structure of SSM can be built in either a centralized or distributed fashion on top of the content and experiential modules. This implies that, at the present, we mainly utilize the computational and software facilities available in participating organizations and UNIX-based operating systems. The relational data model is expected to be used to model course modules. Local data sources can be built on top of various database management systems, ranging from Microsoft Access to PeopleSoft. As a security measure, provisions are made to provide different access capabilities to different classes of users, e.g., students, administrators, or course developers, based on their individual profiles. In addition, to facilitate user access to the system, an interactive, dynamic, and user-friendly graphical user interface (GUI) is being developed. The GUI will guide and direct users to the system, and aid them in finding relevant sources of information based on the keywords they enter and with the help of our metadata. All learning objects developed in the course of the proposed work will adhere to Sharable Content Object Reference Model (SCORM) [24-26], standards to facilitate interoperability with other educational platforms, and the navigational tools will be cognizant of SCORM metadata, to allow query of learning objects external to PERCEPOLIS.

\subsection{Personalization of Course Content and Tracking of Student Progress}

As noted before, we will extend our research in the areas of pervasive computing and mobile agent technology to create a virtual environment that supports a networked curricular model and a subject-based one-to-one student-faculty ratio for the purpose of mentoring, advising, guiding, and educating students within the program. As described earlier in this paper, a student agent, in communication with the agent of his/her academic advisor, in concert with his/her accumulated academic background, interests, and special needs, determines a personalized trajectory of courses and/or modules for the student. In 
this process, the SSM is consulted as a backbone data source to determine the personalized trajectory, as well as a contact course advisor (naturally, in most cases this would be the designated course instructor or course supervisor). At this point, various communication means are set up for the duration of the semester, to facilitate interaction between the student and instructor/advisor.

An agent is associated with each module. The agent is also linked to other agents (modules) in the same course, as well as related modules in other courses. The agents representing various modules within a course form a community among themselves, representing that course. The agent associated with a module reports directly to the instructor, but also serves as a virtual guide to the student for the duration of the course.

More specifically, when a student, $s$, is required to take a course, $c$, an agent, $D_{s c}$, is created to represent the student in that course. $D_{s c}$ acquires information about the student's profile and determines the student's academic background, interests, and accumulated prerequisite knowledge, among other required information. $D_{s c}$ interacts with the agents of course modules to identify a personalized trajectory of modules and learning objects for the student. Furthermore, $D_{s c}$ ensures that the student peruses required material-notes, sample programs, exercises, and the like. $D_{s c}$ also alerts the student to timelines, class schedules, learning/discussion schedules, project deadlines, appointments with the instructor, and corresponding preparations. The instructor agent for the course, $D_{s c}$, ensures that the student meets all requirements for each mandatory module, and collaborates with $D_{s c}$ to ensure that the student is supplied with all required course material. $D_{s c}$ also informs the instructor about the progress of the student and alerts the instructor whenever the student is progressing too slowly or too rapidly.

\section{Anticipated Outcomes}

A significant body of research [2] has concluded that effective learning takes place in the course of a "cognitive apprenticeship" that fosters the learner's intellectual development $[2,27,28]$. Four concepts have been identified as essential to the critical examination and improvement of education; each concept represents a stage in the cognitive apprenticeship:

1) Modeling: enabling students to observe an expert in action.

2) Scaffolding: provide support for students to emulate expert performance.

3) Coaching: providing students with deliberate, planned feedback that guides their performance from novice- to expert-level.

4) Fading: removing the scaffolding as students be- come more competent.

PERCEPOLIS facilitates all four stages of cognitive apprenticeship. In the modeling stage, multimedia demonstrations and animations can be incorporated into the course modules as representations of expert performance. Solved problems, design examples, and guided exercises with "hints" are among the learning objects useful in the scaffolding stage. Online assessments, some of which are graded instantaneously, are examples of learning objects that facilitate coaching. The fine-grained data collection enabled by the cyberinfrastructure facilitates the provision of more accurate and detailed feedback to the students, which is especially helpful in the coaching stage. Graceful and gradual fading of instructor support is also supported, e.g., through learning objects that feature advanced problems or exercises. The availability of online learning objects that can be perused at a student's convenience provides subtle assistance that encourages and supports efforts in the absence of the instructor, further facilitating fading.

PERCEPOLIS enables blended learning, where online, computer-mediated instruction supplements traditional classrooms. Blended learning is an emerging phenomenon well-suited to the learning styles and expectations of today's students, who are digital natives [29]. Students are expected to peruse before class learning objects pertaining to concepts traditionally taught during the lecture portion of a course. This allows class time to be used for problem-based learning and other educational approaches conducive to the cognitive apprenticeship. As an example, the students can be required to peruse a learning object that presents the general steps of a design technique prior to class. In-class demonstrations of the technique on an actual design problem by the instructor or a guest expert can serve as a model for the students. Alternatively, they can work on a design problem in class (scaffolding) and receive feedback (coaching). Gradually increasing the sophistication of the in-class activities supports fading.

The peer and active learning opportunities offered by in-class activities can strengthen the interpersonal skills of the students, facilitate the interaction of students from typically underrepresented groups with their peers, and can be used to incorporate discussions of ethics, sustainability, and other topics beyond the technical knowledge typically conveyed in a traditional lecture setting. Furthermore, a wide range of instructional tools can be utilized to build effective scaffolds for a broad range of "missing basics": reflective and metacognitive skills, interpersonal communication, negotiation, and teamwork. For example, forum tools in existing course management platforms can be used to initiate and scaffold both asynchronous, written group activities (discussion boards) 
and synchronous, live team negotiations (voice boards, online conferencing tools). The wide variety of tools will then address various learning styles used in personalizing the learning trajectory of each student. These activities can be monitored by the instructor, the advanced students serving as peer leaders, or both. The involvement of peer leaders will help them build strong leadership skills, strongly valued by the future employers. On the other hand, participation of the instructor and/or the peer leader in these forums increases their effectiveness and ensures conditions for the fair and ethical behavior requisite of professional practice.

The flexibility of the proposed cyberinfrastructure in utilizing computing technology to support rich educational content has the added benefit of facilitating the accommodation of differences in learning styles. The average learner retains about $20 \%$ of what is heard, $40 \%$ of what is seen and heard, and $75 \%$ of what is seen, heard, and performed [30-32]. A traditional classroom setting mainly offers visual and auditory content; printor video-based distance study does the same. The ability to provide tactile content, whether in reality, in a classroom setting, or through remote access and virtual reality, is especially helpful in the modeling stage of the cognitive apprenticeship, but can also be exploited to increase the efficacy of other stages. Furthermore, the predominant learning style of a student can be assessed and used in personalizing courses and curricular trajectories for each student. The ability to track the perusal of educational content by each student allows more accurate determination of his/her learning style [33].

The dynamic nature of PERCEPOLIS lends itself to a wide range of disciplines beyond science and engineering. As an example, a wealth of rich electronic artifacts have been developed and made available by cultural institutions, e.g., the Metropolitan Museum of Art provides a database of images, audio files, and detailed information about 130,000 works of art in their permanent collection, podcasts, and scientific research information on its web site [34]. An intelligent access tool can allow these resources to be efficiently and dynamically incorporated into classroom discussions and linked to electronic learning objects available for perusal by the students outside of class.

The self-paced and modular nature of the courses and the "anytime, anywhere" availability of the learning objects make the platform well-suited to eLearning, distance education, outreach activities, and workforce education or certificate programs. The same attributes allow graduate students engaged in interdisciplinary research to acquire the requisite broad background without having to complete traditional undergraduate level courses. The software and content artifacts of PERCEPOLIS serve as effective advising tools that can facilitate student course scheduling. Finally, the special accommodations needed for students with learning disabilities or physical challenges are aspects of personalization facilitated by the cyberinfrastructure. Outside of academia, the intelligent personalization facilitated by PERCEPOLIS can be useful to streamlining professional training, whether technical or related to topics such as safety, workplace conduct, or cultural sensitivity.

Beyond the fundamental objective of personalizing the learning trajectory of a student through a networked curriculum, the PERCEPOLIS cyberinfrastructure aims to utilize teaching tools, animation techniques, and remote access to information to present the same information in different ways and accommodate differences in learning styles; allow self-pacing, privacy, and flexibility; achieve spatial and temporal uniformity in presentation of the same material; and ensure efficient utilization of resources and lower costs to students and providers. The following outcomes are anticipated as a result of successful adoption of the proposed cyberinfrastructure.

1) Creation of a dynamic, student-sensitive, and adaptable blended learning environment that allows a virtual one-to-one instructor-to-student ratio.

2) Definition of a novel methodology for modular course development, implementation, and delivery that promotes continuous, self-paced, active, and peer learning and supports networked curricula.

3) Development of a novel navigational tool for student advising and course management that facilitates access, browsing, development, expansion, and modification of curricula and courses across multiple disciplines and institutions.

4) Development of a new technology-based methodology for effective and efficient presentation of courses and active engagement of students in the classroom.

5) A shift to professional practice as the focus of educational efforts, rather than technical skills.

The project is currently in the proof-of-concept stage. We are in the process of converting the following four courses to the modular format required for use in PERCEPOLIS: Introduction to Computer Engineering, Digital System Design, Computer Organization and Design, and Introduction to High Performance Computer Architecture.

The educational research goals of the project are the following, inspired by the Technology-Enabled Active Learning (TEAL) project at the Massachusetts Institute of Technology [35].

1) In the cognitive domain, to assess students' mastery of concepts as a result of studying in courses where the PERCEPOLIS platform is utilized - does the personalization with respect to background and learning style 
help or hinder student learning?

2) In the affective domain, to analyze students' attitudes towards the blended personalized learning environment, and to study their preferences regarding the combination of various teaching methods and learning objects - is personalization an effective support for students' learning, as well as an efficient instructional strategy in the modularized blended learning environment?

3) In the social domain: to characterize the effect of using the PERCEPOLIS platform on student interactions, both with the instructor and with their peers, and to assess soft skills such as communication and negotiation does the proposed platform support high-impact strategies to enhance the students' ability to build the soft skills valued by future employers?

Expected measurable outcomes include: their concerns and questions with their peers.

- Success of personalization (cognitive, affective)

- Effectiveness of teaching practice (cognitive, affective, social)

- Interconnection among different courses - module reuse (cognitive, social)

- Student satisfaction, as compared to traditional advising - can use dropout or change of major as a gauge (affective)

- Employer satisfaction with soft and technical skills (social, cognitive)

The novelty of PERCEPOLIS lies in its ability to leverage pervasive and ubiquitous computing and communication through the use of intelligent software agents that use a student's academic profile and interests, as well as supplemental information such as his/her learning style, to customize the content of a course for the student. Furthermore, PERCEPOLIS facilitates the collection of data on student performance and learning at a resolution that far exceeds what is currently available. Knowledge discovery from this rich data set can yield invaluable insights, such as the efficacy of particular instructional techniques and strategies embedded in the learning objects. The data collected is also helpful for accreditation self-studies. Learning objects, in particular assessments can be linked to specific learning outcomes of a course.

\section{Conclusions}

The proposed cyberinfrastructure supports a shift from teaching technical skills to fostering the professional expertise required for a student to succeed as a practicing engineer. The dynamic nature of PERCEPOLIS lends itself to a wide range of disciplines beyond engineering. The self-paced and modular nature of the courses and the "anytime, anywhere" availability of the learning objects make the platform well-suited to eLearning, distance education, outreach activities, and workforce education or certificate programs. The same attributes allow graduate students engaged in interdisciplinary research to acquire the requisite broad background without having to complete traditional undergraduate level courses. The software and content artifacts of PERCEPOLIS serve as effective advising tools that can facilitate student course scheduling. Finally, the special accommodations needed for students with learning disabilities or physical challenges are aspects of personalization facilitated by the cyberinfrastructure.

\section{References}

[1] B. York, A. van Dam, J. Ullman, E. Soloway, J. Pollack, A. Kay and T. Kalil, "A Teacher for Every Learner: Scalable Learner-Centered Systems,” 2010. http://www. nesc.ac.uk/esi/events/Grand_Challenges/usa/education.pdf

[2] S. D. Sheppard, K. Macatangay, A. Colby and W. M. Sullivan, "Educating Engineers: Designing for the Future of the Field," Carnegie Foundation for the Advancement of Teaching, Jossey-Bass, USA, 2009.

[3] National Academy of Engineering, "Grand Challenges for Engineering,” 2010. http://www.engineeringchallenges.org, accessed Jan

[4] M. Satyanarayanan, "Pervasive Computing: Vision and Challenges,” IEEE Personal Communications, Vol. 8, No. 4, 2001, pp. 10-17.

[5] G. Banavar and A. Bernstein, "Software Infrastructure and Design Challenges for Ubiquitous Computing Applications," Communications of the ACM, Vol. 45, No. 12, 2002, pp. 92-96.

[6] D. Lange and M. Oshima, "Programming and Developing Java Mobile Agents with Aglets,” Addison-Wesley, New York, 1998.

[7] R. Gray, D. Kotz, G. Cybenko and D. Rus, "Mobile Agents: Motivations and State-of-the-Art Systems,” In: J. Bradshaw, Ed., Handbook of Agent Technology, AAAI/ MIT Press, Cambridge, 2002, pp. 1-30.

[8] A. R. Hurson, E. Jean, M. Ongtang, X. Gao, Y. Jiao and T. E. Potok, "Recent Advances in Mobile Agent-Oriented Applications,” In: L. Yang and A. B. Waluyo, Eds., Mobile Intelligence: When Computational Intelligence Meets Mobile Paradigm, John Wiley \& Sons, New York, 2009, pp. 106-139.

[9] Y. Jiao and A. Hurson, "Performance Analysis of Mobile Agents in Heterogeneous Mobile Database Systems," Proceedings of the IEEE International Conference on Emerging Technologies (ICET'03), Minneapolis, USA, 2003.

[10] Y. Jiao and A. R. Hurson, "Mobile Agents in Mobile Data Access Systems," Proceedings of the 10th International Conference on Cooperative Information Systems (COOPIS), Irvine, 2002, pp. 144-162.

[11] E. Jean, Y. Jiao and A. R. Hurson, "Addressing Mobile Agent Security through Agent Collaboration,” Journal of 
Information Processing Systems, Vol. 3, No. 2, 2008, pp. 43-53.

[12] M. Ongtang, A. R. Hurson and Y. Jiao, “Agent-Based Infrastructure for Data and Transaction Management in Mobile Heterogeneous Environment," Proceedings of the WRI International Conference on Communications and Mobile Computing (CMC’09), Yunnan, 2009, pp. 314318.

[13] E. Jean, R. T. Collins, A. R. Hurson, S. Sedigh and Y. Jiao, "Pushing Sensor Network Computation to the Edge," Proceedings of the 5th International Conference on Wireless Communications, Networking and Mobile Computing (WiCOM '09), Beijing, China, 2009, pp. 1-4.

[14] S. Sedigh, A. R. Hurson and B. Shirazi, "Tools and Techniques for Interoperability and Dynamic Reconfiguration of Pervasive Systems,” In: M. Denko and M. S. Obaidat, Eds. Pervasive Computing and Networking, John Wiley \& Sons, New York, 2010.

[15] M. W. Bright, A. R. Hurson and S. H. Pakzad, "A Taxonomy and Current Issues in Multidatabase Systems," Computer, Vol. 25, No. 3, 1992, pp. 50-60.

[16] M. W. Bright, A. R. Hurson and S. H. Pakzad, “Automated Resolution of Semantic Heterogeneity in Multidatabases," ACM Transactions on Database Systems, Vol. 19, No. 2, 1994, pp. 212-253.

[17] M. W. Bright, A. R. Hurson, S. Pakzad and H. Sarma, "The Summary Schemas Model-An Approach for Handling Multidatabases: Concept and Performance Analysis," Multidatabase Systems: An Advanced Solution for Global Information Sharing, 1999, pp. 199-216.

[18] B. Yang and A. R. Hurson, "Semantic-Aware and QoS-Aware Image Caching in Ad Hoc Networks,” IEEE Transactions on Knowledge and Data Engineering, Vol. 19, No. 12, 2007, pp. 1694-1707.

[19] J. B. Lim, A. R. Hurson and Y. Jiao, "Transaction Processing in Mobile, Heterogeneous Database Systems," Encyclopedia of Computer Science and Engineering, Vol. 5, 2008, pp. 2922-2930.

[20] K. Dash, A. Hurson, S. Phoha and C. Chehadeh, "Summary Schemas Model: A Scheme for Handling Global Information Sharing," Proceedings of the International Conference on Intelligent Information Management Systems, Washington, DC, 1994, pp. 47-51.

[21] L. Smith and A. Hurson, "A Search Engine Selection Methodology," Proceedings of the International Conference on Information Technology: Coding and Computing (ITCC’03), Las Vegas, NV, 2003, pp. 122-129.

[22] A. R. Hurson and M. W. Bright, "Multidatabase Systems: An Advanced Concept in Handling Distributed Data,"
Advances in Computers, Vol. 32, 1991, pp. 149-200.

[23] J. B. Lim and A. R. Hurson, "Transaction Processing in Mobile, Heterogeneous Database Systems," IEEE Transactions on Knowledge and Data Engineering, Vol. 14, No. 6, 2002, pp. 1330-1346.

[24] I. Kazanidis and M. Satratzemi, "Efficient Authoring of SCORM Courseware Adapted to User Learning Style: The Case of ProPer SAT," Proceedings of the International Conference on Advances in Web-Based Learning (ICWL '09), Germany, 2009, pp. 196-205. http://www. springerlink.com/content/7x421jm53n885t53

[25] Advanced Distributed Learning Initiative, "Sharable Content Object Reference Model (SCORM),” 2010. http:// www.adlnet.org

[26] Learning Systems Architecture Laboratory, "SCORM Best Practices Guide for Content Developers,” Technical Report, Carnegie Mellon University, 2005. http://www. jointadlcolab.org/downloads/scormcdg1edsec7update 200 50420.pdf

[27] A. Collins, J. S. Brown and S. E. Newman, "Cognitive apprenticeship: Teaching the Crafts of Reading, Writing, and Mathematics,” Knowing, Learning, and Instruction: Essays in Honor of Robert Glaser, 1989, pp. 453-494.

[28] J. S. Brown, A. Collins and P. Duguid, "Situated Cognition and the Culture of Learning," Educational Researcher, Vol. 18, No. 1, 1989, p. 32.

[29] G. Hislop, “The Inevitability of Teaching Online,” Computer, Vol. 42, No. 12, 2009, pp. 94-96.

[30] R. Felder and L. Silverman, "Learning and Teaching Styles in Engineering Education," Engineering Education, Vol. 78, No. 7, 1988, pp. 674-681.

[31] S. Reisman and W. A. Carr, "Perspectives on Multimedia Systems in Education," IBM Journal of Research and Development, Vol. 30, No. 3, 1991, pp. 280-295.

[32] T. Yager, “Information's Human Dimension,” Byte Magazine, Vol. 16, No. 13, 1991, pp. 153-160.

[33] E. Popescu, "Evaluating the Impact of Adaptation to Learning Styles in a Web-Based Educational System," Proceedings of the 8th International Conference on Advances in Web Based Learning (ICWL '09), Germany, 2009, pp. 343-352.

[34] “The Metropolitan Museum of Art," 2010. http://www. metmuseum.org/works of art

[35] Y. J. Dori and J. Belcher, "How Does Technology-Enabled Active Learning Affect Undergraduate Students' Understanding of Electromagnetic Concepts?" Journal of the Learning Sciences, Vol. 14, No. 2, 2005, pp. 243-279. 